\title{
Correspondence
}

\section{IV fentanyl PCA during labour}

To the Editor:

The clinical report by Rosaeg et al. ${ }^{\prime}$ identifies correctly the usefulness of intravenous PCA with fentanyl for the provision of analgesia during labour when epidural analgesia is contraindicated. The authors report that during the accelerated first stage of labour $(7-10 \mathrm{hr}$ after the start of PCA fentanyl), coinciding with the augmentation of labour with intravenous oxytocin, the patient had inadequate pain relief and lost confidence in the ability of the PCA pump (and perhaps the anaesthetist supervising the PCA) to give her satisfactory analgesia. They do not mention any alterations made to the programming of the PCA pump in response to the obvious inadequacy of the current parameters. In fact, the analgesic demand histograms and the text suggest that this state was allowed to continue for several hours.

If this patient had been considered an appropriate candidate for and had received epidural analgesia with inadequate pain relief in the late first stage of labour, would the authors have intervened or allowed her pain to continue unabated? We assume they would offer a "topup," increase the continuous infusion, or replace the epidural catheter if appropriate. Why then should they treat a patient receiving iv PCA differently?

The pharmacodynamic variability in analgesic requirements during labour is marked. Analgesic requirements will almost certainly increase as the intensity and severity of painful stimuli (contractions) increase when the parturient enters the late first stage of labour. If iv PAC therapy is to be effective, it is important that the nurse assess the patient's response to the analgesic regimen, and the pump's memory (answered vs. unanswered demands) be reviewed at frequent intervals. The physician supervising the iv PCA therapy must be prepared to make appropriate changes as required. The use of empirically derived "cookbook recipes" without appropriate follow-up or intervention is unacceptable.

In our experience with the use of iv PCA for labour analgesia, we have found it is often necessary to alter one or all of the programming variables which include the background infusion, the amount of the bolus dose, the duration of the lockout interval, or even provide an additional loading dose to optimize analgesia.

IV PCA is an innovative analgesic system that is useful for relieving pain in labour. In selected cases, it has been demonstrated to be safe and effective. However, to use PCA successfully in the labouring parturient, the anaesthetist should be familiar with the concept and principles of PCA and its practical applications.

Robin J. Gavelin MD FRCPC

James A. Janzen MD FRCPC

Foothills Provincial General Hospital

Calgary, Alberta

\section{REFERENCE}

1 Rosaeg $O P$, Kitts JB, Koren G, Byford LJ. Maternal and fetal effects of intravenous patient-controlled fentanyl analgesia during labour in a thrombocytopenic parturient. Can J Anath $1992 ; 39 ; 277-81$.

\section{REPLY}

In their letter, Drs. Gavlin and Janzen expressed concern at the apparent lack of satisfactory analgesia during the first stage of labour, and questioned why we might treat a parturient receiving iv PCA fentanyl differently from a patient receiving epidural analgesia for labour. There are several reasons. It is reported that epidural analgesia is both safe and efficacious when administered to control labour pain. The literature does not contain publications which document the safety or efficacy of iv PCA fentanyl when administered to control labour pain.' Our literature search provided a single case report, where the parturient received a total dose of $400 \mu \mathrm{g}$ of fentanyl during labour. ${ }^{2}$ In the management of our patient, we approached a total dose of $1.0 \mathrm{mg}$ fentanyl. Due to the lack of data determining maternal and fetal safety with iv PCA fentanyl during labour and without additional monitoring precautions we were reluctant to provide unlimited drug to achieve "satisfactory analgesia." In addition, most anaesthetists have a wealth of experience in providing epidural analgesia while very few have any experience with iv PCA fentanyl during labour. Finally, comparing the efficacy of epidural local anaesthetics for pain relief during labour with iv PCA fentanyl during labour is unrealistic. Epidural local anaesthetics are successful in abolishing labour pain in most cases, while iv PCA fentanyl, even in appropriate does, is unlikely to provide similar pain relief.

We agree with Drs. Gavelin and Janzen that the pharmacodynamic variability in analgesic requirements during labour is marked. The advantage of iv PCA is to allow the patient to achieve an analgesic serum concentration level that is specific to the individual. The very concept of PCA allows titration of drug to individual requirements which is contradictory to a "cook-book recipe" approach.

We emphasized in our report that further objective data from controlled clinical studies are required to document safery (maternal and fetal) and efficacy of iv PCA fentanyl when administered during labour. Although Drs. Gavelin and Janzen state that they optimize analgesia by changing the various pump 
variables and increasing the bolus dose in the parturient, they do not mention any adverse effects, matemai or fetal. Unforturateby, we did not have access to their anecdotal experience to guide our management of this patient. Therefore, we encourage Drs. Gavelin and Janzen to publish their data so that the technique of iv PCA fentanyl administration for the parturient can be improved.

Ola P. Rosaeg MB FRCFC

John B. Kitts MD FRCPC

Ottawa Civic Hospital

Ottawa, Ontario

\section{REFERENCES}

1 Douglas $M J$. Alternatives to epidural analgesia during labour. Can J Anaesth 1991; 38: 421-4.

2 Klelman SJ, Wiesel S, Tessier MJ. Patient-controlled analgesia (PCA) using fentanyl in a parturient with a platelet function abnormality. Can J Anaesth 1991; 38: 489-91.

\section{Obstetric anaesthesia and spinal cord injury}

\section{To the Editor:}

We enjoyed reading the review by Crosby et $a l^{1}$ on the management of obstetrical patients with spinal cord injuries. These authors provided a complete analysis of the pathophysiology and risks of these patients during labour and delivery, with emphasis on the prevention and treatment of autonomic hyperreflexia. However, there was no discussion of the potential airway management problems in these patients. We recently cared for a woman during labour and delivery with Kugelberg-Welander disease, a form of spinal muscle atrophy. Although not a spinal cord injury, she suffered from progressive spinal cord weakness and deterioration, and in all respects had the same implications for anaesthetic management, except for the potential for autonomic hyperreflexia.

We were asked to provide anaesthesia for primary Caesarean section for this 34 -yr-old, $G_{1} P_{0}$, hispanic female who presented at $37 \mathrm{wk}$ gestation in active labour. She had a history of progressive spinal muscle atrophy since birth. She had several hospital admissions during her pregnancy for treatment of pneumonia. Her past surgical history included placement of Harrington rods (cervical through lumbar) and cholecystectomy under general anaesthesia without complication. She suffered frequent bouts of asthma. Her medications included penicillin, iron, nasal cannula ox ygen prn, and inhaled albuterol prn. Pulmonary function tests five months earlier revealed FVC $1.03 \mathrm{~L}$ ( $31 \%$ predicted) and a FEV $0.89 \mathrm{~L}$ (34\% predicted). Vaginal delivery was considered not to be possible, because of cephalopelvic disproportion, and her weak abdominal and pelvic musculature.
On physical examination, the patient was unable to lie supine and there was severe limitation of neck movement, confined to the neutral position. Her tongue was large and the uvula could not be visualized.

The patient was premedicated with intravenous metaclopramide $10 \mathrm{mg}$, and $30 \mathrm{ml}$ of oral sodium citrate solution. Nasotracheal intubation was preferred, after topical anaesthesia with the help of a flexible fibreoptic endoscope. General anaesthesia was induced with thiopentone and maintained with isoflurane, and a healthy male infant was delivery uneventfully. At the completion of surgery, the patient was transferred to the surgical intensive care unit. She received positive-pressure ventilation, optimization of her bronchodilators and pulmonary toilet, and the trachea was extubated without difficulty the following day. She was discharged home one week later.

We were faced with the problem of providing anaesthesia for this woman with marginal pulmonary reserve, who could not lie supine, nor move her neck from the neutral position. Epidural anaesthesia was considered but rejected because of her previous back surgery. Spinal anaesthesia was undesirable due to lack of precise controllability. Either may have exacerbated respiratory muscle weakness.

Our case illustrates the difficulties anaesthetists may encounter when managing the airway of spinal cordinjured obstetric patients. The problems are considerable and should be addressed prospectively. After considering the various methods for anaesthesia, the safest, most conservative approach should be utilized.

Ronald S. Litman DO
Rodney Voisine MD
Rochester, N.Y.

REFERENCE

1 Crosby E, St-Jean B, Reid D, Elliott RD. Obstetrical anaesthesia and analgesia in chronic spinal cord-injured women. Can J Anaesth 1992; 39: 487-94.

\section{Malignant hyperthermia susceptibility in patients with Duchenne's muscular dystrophy}

To the Editor:

Duchenne's muscular dystrophy (DMD) is a severe and progressive form of myopathy that is transmitted by an $X$ linked recessive gene with symptoms becoming apparent in males between the ages of two and six years. There are conflicting reports regarding the susceptibility of patients 